\title{
Price Prediction of Stock Market using Hybrid Model of Artificial Intelligence
}

\author{
Mohammad Badrul Alam \\ Miah \\ Dept. of Information and \\ Communication Technology \\ Mawlana Bhashani Science \\ and Technology University \\ Santosh, Tangail-1902, \\ Bangladesh
}

\author{
Md. Zakir Hossain \\ Dept. of Information and \\ Communication Technology \\ Mawlana Bhashani Science \\ and Technology University \\ Santosh, Tangail-1902, \\ Bangladesh
}

\author{
Md. Amjad Hossain \\ Dept. of Information and \\ Communication Technology \\ Mawlana Bhashani Science \\ and Technology University \\ Santosh, Tangail-1902,
}

Bangladesh

\author{
Md. Muzahidul Islam \\ Dept. of Information and Communication Technology \\ Mawlana Bhashani Science and Technology University \\ Santosh, Tangail-1902, Bangladesh
}

\begin{abstract}
Today, investment by purchasing stock-share constitutes the greater part of economic exchange of countries and a considerable amount of capital is exchanged through the stock markets in the whole world. But one of the most important problems is finding efficient ways to summarize and visualize the stock market data to individual or institutions useful information about the market behavior for investment decision. This research proposes an intelligent stock market forecasting system using the ability of neural network and fuzzy inference system to discover patterns in nonlinear and chaotic systems. This research with a probe in a sample of the whole population of the study involves the data financial record of BEXIMCO Ltd. which is member of Chittagong stock exchange, Bangladesh, aims at the prediction of stock price. The prediction was done by a nonlinear fuzzy-neural network model using exogenous variable of stock market.
\end{abstract}

\section{General Terms}

Artificial Intelligence, Artificial Neural Network, Machine Learning, Back Propagation Algorithms, Fuzzy Inference System, Stock Market.

\section{Keywords}

Artificial Intelligence (AI), Artificial Neural Network (ANN), Feed Froward Network, Prediction, Back Propagation, Fuzzy Logic, Stock Market, and Share.

\section{INTRODUCTION}

The stock market is always one of the most popular investments due to its high profit. The ability to predict the stock price to meet the fundamental needs of investors and market makers for gaining more financial benefits is an important subject. This issue has attracted lots of audiences among the economic societies. Stock markets are influenced by many factors and this feature has created a highly unreliability in this field. Price changes is a small index of the whole market data, as financial markets are always tied with social events, by only considering price schemes we cannot reach a suitable prediction in the stock price.

Researchers have used a variety of means and models for these purposes. Among them we can mention technical and empirical methods and predictions in the time series and methods based on intrinsic value of stock share and models based on artificial intelligence in the derivation of dominative patterns of the stock [1].

This paper demonstrates a hybrid model of Artificial Intelligence which includes Artificial Neural Network and Fuzzy Inference System. Back propagation method for training the Neural Network and Multilayer Feed forward network in order to forecast the share values and Fuzzy Inference system in order to validate that predicted values. The aim of this paper is to use the power of ANNs and Fuzzy logic in a combination to forecast Chittagong Stock Exchange market index values with reasonable a degree of accuracy.

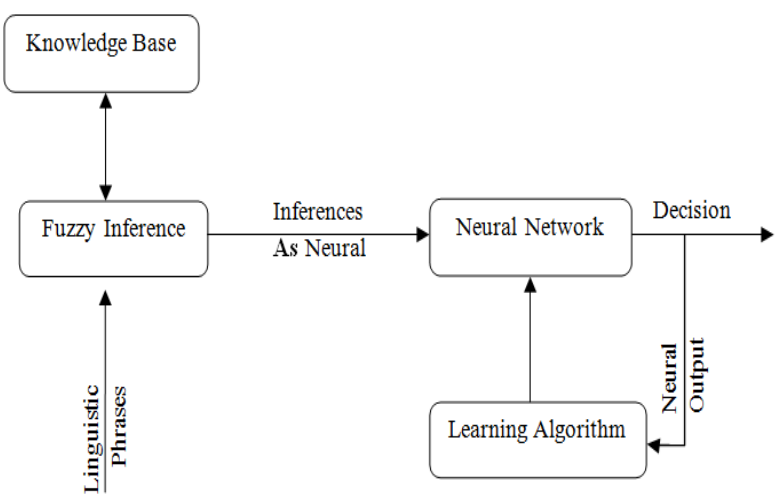

Fig 1: The basic structure of the Fuzzy-neural system.

\section{PREDICTION METHODOLOGY ANALYSIS}

Before the last few decades people traded stocks and commodities primarily on intuition. As the level of investing and trading grew, people searched for tools and methods that would increase their gains while minimizing their risk. Statistics, technical analysis, fundamental analysis, and linear regression are all used to predict and benefit from the market's direction. None of these techniques has proven to be the consistently correct prediction tool that is desired, and many analysts argue about the usefulness of many of the approaches. However, these methods are presented as they are commonly used in practice and represent a base-level standard for which artificial intelligence should outperform. Also, many of these techniques are used to preprocess raw data inputs. 


\subsection{The Efficient Market Hypothesis}

The Efficient Market Hypothesis (EMH) states that at any time, the price of a share fully captures all known information about the share [2]. Since all known information is used optimally by market participants, price variations are random, as new information occurs randomly. Thus, share prices perform a "random walk", and it is not possible for an investor to beat the market.

The EMH is important because it contradicts all other forms of analysis. If it is impossible to beat the market, then technical, fundamental, or time series analysis should lead to no better performance than random guessing [2]. The fact that many market participants can consistently beat the market is an indication that the EMH may not be true in practice. The EMH may be true in the ideal world with equal information distribution, but today's markets contain several privileged players who can outperform the market by using inside information or other means.

\subsection{Chaos Theory}

A relatively new approach to modeling nonlinear dynamic systems like the stock market is chaos theory. Chaos theory analyzes a process under the assumption that part of the process is deterministic and part of the process is random. Chaos is a nonlinear process which appears to be random [2, 3]. Various theoretical tests have been developed to test if a system is chaotic (has chaos in its time series). Chaos theory is an attempt to show that order does exist in apparent randomness. By implying that the stock market is chaotic and not simply random, chaos theory contradicts the EMH.

In essence, a chaotic system is a combination of a deterministic and a random process [2]. The deterministic process can be characterized using regression fitting, while the random process can be characterized by statistical parameters of a distribution function. Thus, using only deterministic or statistical techniques will not fully capture the nature of a chaotic system. A neural networks ability to capture both deterministic and random features makes it ideal for modeling chaotic systems.

\subsection{Other Computer Techniques}

Many other computer based techniques have been employed to forecast the stock market. They range from charting programs to sophisticated expert systems. Fuzzy logic has also been used.

Expert systems process knowledge sequentially and formulate it into rules [4]. They can be used to formulate trading rules based on technical indicators. In this capacity, expert systems can be used in conjunction with neural networks to predict the market. In such a combined system, the neural network can perform its prediction, while the expert system could validate the prediction based on its well-known trading rules [2].

\section{ISSUES IN PREDICTION OF SHARE MARKET PRICE}

The main problem in predicting share market is that the share market is a chaos system. There are many indicators that could affect the share market directly or indirectly. There are no significant relations between the indicators and the price. We cannot draw any mathematical relation among the indicators. There are no hard and fix rules of predicting the share price using these indicators.

\section{OUR SYSTEM MODEL}

For this kind of chaotic system the neural network and fuzzy logic together is suitable because we do not have to understand the solution. This is a major advantage of Fuzzyneural system [5]. On the other hand in the traditional techniques we must understand the inputs, the algorithms and the outputs in great detail.

With the Fuzzy-neural system we just need to simply show the correct output for the given inputs. With sufficient amount of knowledge base and training, the system will mimic the function $[5,6]$. Another advantage of this system is that during the tanning process, the network will learn to ignore any inputs that don't contribute to the output $[5,6]$.

In our system, there are two parts one is Fuzzy Inference part and the other is Neural Network part. In the Fuzzy Inference part we have used two major indicators that have most significant effect on future stock price, and each indicator has nine rules in combined. By this part we get the target stock price which is used in the Neural Network part. On the other hand in the Neural Network part there is a training phase where some indicators named weights are used that have significant effect on the future stock price. These weights are used in prediction phase using same equations which are used in training phase. Backpropagation Algorithm is used for the training phase. This is our basic architecture of our System and this approach is known as a Fuzzy-neural system. There are a lot of indicators in share market which have impacts in future share price. But all the indicators are not used in our system because their impacts are not equally significant in share market price. We used 6 inputs for the system. The inputs are: General Index (GI), Market Capitalization (MC), Price Earning (P/E ) ratio, Net Asset Value (NAV), Earnings per Share (EPS) and volume. Then we create a knowledge base using these data and normalized the data set according to the network and the feed the data to the network.

\subsection{The Fuzzy Logic Model}

The collected stock factors are always associated with some error, which makes the fuzzy approach more suitable [7]. First of all, the fuzzy approach provides possible rules relating input variables to the output variable; hence, it is more in-line with human thought. Therefore, stock investors can rapidly develop their own set of rules to test for their fit for the fuzzy model.

Fuzzy logic rules are verbal expressions in "IF-THEN" format like IF low AND very low THEN hot [8]. For the model of this study 2 input variables General Index (GI), Market Capitalization (MC), and one output (Target Close Price) parameters were used. The aim was to create such rules that will relate 8 inputs with the output verbally using 9 member functions of each. For this purpose, the Target Close Price is forwarded to the neural network model, who uses this as target input during training the network.

\subsection{Backpropagation with Feedforeword NN}

Back-propagation algorithm $[4,9,10]$ is basically the process of back-propagating the errors from the output layers towards the input layer during training sessions. Back-propagation is necessary because the hidden units have no target values which can be used, so these units must be trained based on errors from the previous layers. The output layer has a target value which is used to compare with calculated value. As the errors are back- 
propagated through the nodes, the connection weights are continuously updated. Training will occur until the errors in the weights are adequately small to be accepted. On the other hand the computational complexity of Back-propagation Algorithm is only $\mathrm{O}(\mathrm{n})$. These features of the algorithm are the main criteria for predicting share prices accurately. The main steps using the Backpropagation algorithm as follows:
Step 2: Compute the error between the output(s) and the actual target(s);

Step 3: The connection weights and membership functions are adjusted;

Step 4: IF Error > Tolerance THEN go to Step 1 ELSE stop.

Step 1: Feed the normalized input data sample, compute the corresponding output;

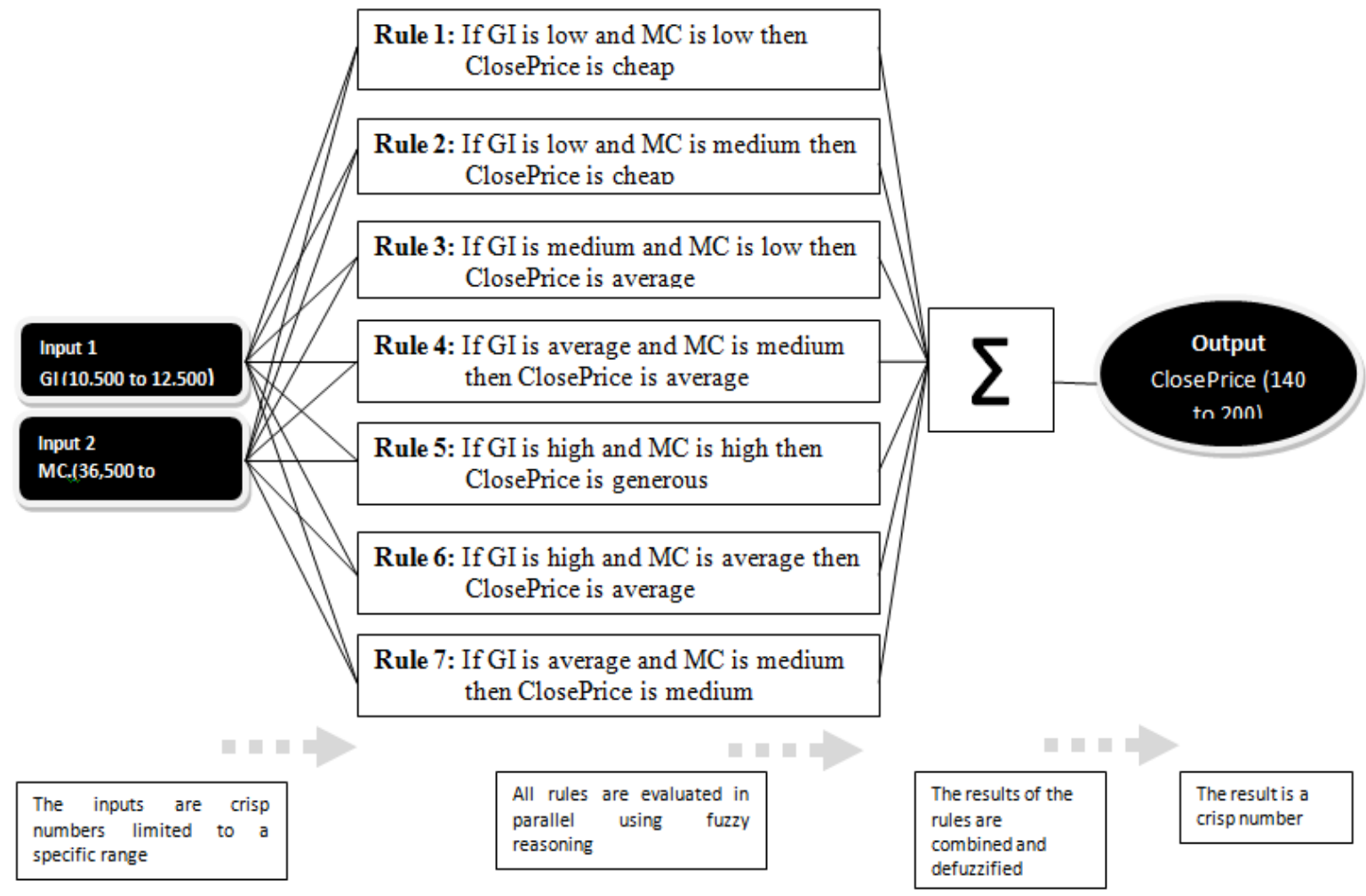

Fig 2: Schematic Diagram for Fuzzy Inference System (FIS)

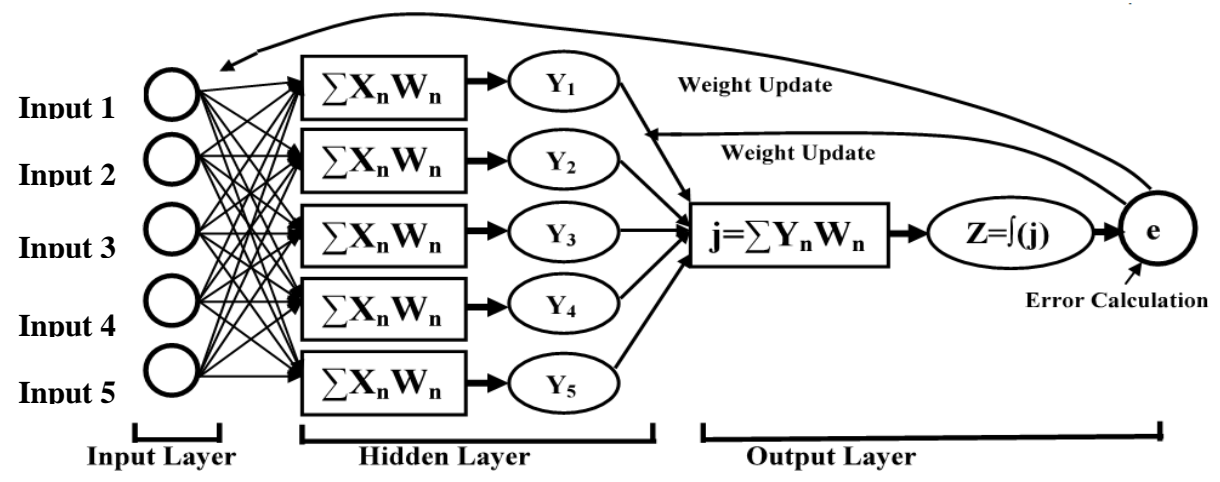

Fig 3: Training phase

\section{SYSTEM ANALYSIS}

In our system there are two basic parts one is Fuzzy Inference part and the other is Neural Network part. Analysis of each part is given below.

\subsection{Knowledge Base Creation of Fuzzy Interference}

In this study, nine membership functions ( $\mathrm{mfs}$ ) were used to create a knowledge base. eight for inputs and one for the output. The numbers of subsets were selected for each $\mathrm{mf}$ using the range for each parameter. Since the number of fuzzy rules is obtained by multiplication of all subsets of input mfs, increasing the number of subsets for inputs would make the rule creation stage of modeling impractical [18 from document.doc] The Fuzzy Inference system gives a target close price to the Neural Network and is used in training of the neural network. 
Table 1: Predicting price, Actual price and Error of BEXIMCO Ltd. Company using 9 input data sets

\begin{tabular}{|c|c|c|c|}
\hline Date & $\begin{array}{c}\text { Actual Price } \\
\text { (Taka) }\end{array}$ & $\begin{array}{c}\text { Predicted Price } \\
\text { (Taka) }\end{array}$ & $\begin{array}{c}\text { Error } \\
(\%)\end{array}$ \\
\hline $\begin{array}{c}\text { 01-January- } \\
\text { 2012 }\end{array}$ & 116.20 & 114.3276 & 1.6114 \\
\hline $\begin{array}{c}\text { 02-January- } \\
\text { 2012 }\end{array}$ & 115.00 & 113.9808 & 0.8863 \\
\hline $\begin{array}{c}\text { 03-January- } \\
\text { 2012 }\end{array}$ & 116.00 & 114.9175 & 0.9332 \\
\hline $\begin{array}{c}\text { 04-January- } \\
\text { 2012 }\end{array}$ & 119.20 & 113.3717 & 4.8895 \\
\hline $\begin{array}{c}\text { 05-January- } \\
\text { 2012 }\end{array}$ & 115.60 & 114.9512 & 0.5612 \\
\hline $\begin{array}{c}\text { 06-January- } \\
\text { 2012 }\end{array}$ & 113.00 & 114.5759 & 1.3946 \\
\hline $\begin{array}{c}\text { 07-January- } \\
\text { 2012 }\end{array}$ & 111.00 & 112.1511 & 1.0370 \\
\hline $\begin{array}{c}\text { 08-January- } \\
\text { 2012 }\end{array}$ & 111.00 & 111.4145 & 0.3734 \\
\hline $\begin{array}{c}\text { 09-January- } \\
\text { 2012 }\end{array}$ & 109.00 & 109.4808 & 0.4411 \\
\hline $\begin{array}{c}\text { 10-January- } \\
\text { 2012 }\end{array}$ & 102.00 & 103.3241 & 1.2981 \\
\hline $\begin{array}{c}\text { 11-January- } \\
\text { 2012 }\end{array}$ & 106.00 & 109.9704 & 3.7457 \\
\hline $\begin{array}{c}\text { 12-January- } \\
\text { 2012 }\end{array}$ & 98.00 & 100.8887 & 2.9477 \\
\hline 13-January- & 102.92 & 99.0232 & 3.7862 \\
\hline
\end{tabular}

\subsection{Training Phase of Neural Network}

There are two phases 1st is the training phase and 2nd is the prediction phase. The training phase can be divided into two parts, the propagation phase and the weight update phase.

In the propagation phase 1 st the input data is normalized for feeding the network into the input nodes using the formula:

$$
v^{\prime}=\frac{v-\min A}{\max A-\min A}\left(n e w_{\max A}-n e w_{\min A}\right)+n e w_{\min A}
$$

Here,

$\mathrm{V}^{\mathrm{ee}}=$ Normalized Input

$\mathrm{V}=$ Actual Input.

Min A, Max A = Boundary values of the old data range. New $\min A$, New maxA = Boundary values of the new data range. In this case it is -1 and 1 because the backpropagation can only handle data between -one to one [2]

From the figure 3 we can see that, the normalized input data are fed into the input layer, then the weights are multiplied with the each input data and enter into the neurons of hidden layer, in our model we used single hidden layer. In our model the hidden layer neurons has the same functions as the input layers neurons. After that each neuron passes the output to the next neuron of the output layer. The output layer calculate the in the same way as the

The above process is done in every weight matrix in the network for updating weight. The Phase 1 and Phase 2 procedure repeatedly used until the sum of square error is zero or close to zero. Each neuron is composed of two units. First unit adds products of weights coefficients and input signals [2]. Then this output enter into the second unit of the neuron which contains the nonlinear activation function, in our model we use sigmoid function as our activation function [11]. The formula of sigmoid activation is: $f(\mathrm{x})=(1 /$ $\left.\left(1+\mathrm{e}^{\text {-input }}\right)\right)$

\subsection{The Prediction Phase}

When the neural network is trained then it is ready for prediction. After training with acceptable error the weights are set into the network then we give the trained network the input data set of the day which price we want to predict. The trained network then predicts the price using the given input data set. hidden layer neuron and generate the final out put which is the compared with the real output and calculate an error signal "e".

$\mathrm{e}=(($ actual output - predicted output $) /$ actual output $) * 100$.

The error "e" is generated from the Propagation Phase is used to update the weight using the following formula:

Updated Weight $=$ weight $($ old $)+$ learning rate $*$ output error* output (neurons i) * output (neurons $\mathrm{i}+1) *(1$ - Output (neurons $\mathrm{i}+1))$.

\section{FACTORS OF STOCK MARKET}

Here is a brief description about the inputs that affect the share price:

\subsection{Demand and Supply}

When people are buying more stocks, then the price of that particular stock increases. On the other hand if people are selling more stocks, then the price of that stock falls. So, you should be very careful when you decide to invest in the Bangladeshi stock market. It is also known as turnover [12].

\subsection{Market Cap}

Never try to guess the worth of a company simply by comparing the price of the stock. You should always keep in mind that it is not the stock but the market capitalization of the company that determines the worth of the company. So market cap is another factor that affects stock price [12].

\subsection{General Index (GI)}

General index is a number that measure the relative value of a section of share market. It reflects the total economic condition of the market. If the general index goes down then it means the economic condition of that particular market is relatively in poor condition [12]

\subsection{Net Asset Value}

The Net asset value (NAV) of a company is the company's total assets minus its total liabilities. NAV is typically calculated on a per-share basis [12].

$\mathrm{NAV}=($ Net asset of a company - Liability $) /$ Total number of outstanding share 


\subsection{Earnings per Share (EPS)}

Earnings per share (EPS) are a comparison tool between two companies. Earnings per share serve as an indicator of a company's profitability [12].

EPS $=$ Net Earnings $/$ Number of Outstanding Shares

\subsection{Share Volume}

Share volume can be calculated in two different types the daily share volume and the monthly share volume. The total number of share is sold in a particular day is called daily share volume. In monthly share volume is the sum of the trading volumes during that month [12].

\section{SIMULATION AND PERFORMANCE ANALYSIS}

By using the past historical data of BEXIMCO Ltd which include 9 inputs, we tried to predict stock values for future 13 days of January 2012 from Back-Propagation algorithm we are now able to compare the predicted values with the real values. Table 1 and Figure 4 show the prediction and real values of the BEXIMCO Ltd. The input past historical data is from 01-02-2011 to 31-12-2011. The average error of the simulation was 1.84 percent.

\subsection{Observation}

The stock price is always associated with a set of factors and the factors are divided into two categories, quantitative factors and qualitative factors. From the above experiment we show that the proposed intelligent stock market forecasting system is able to handle both quantitative and qualitative factors. Such measurements never provide the same exact predicted price after repeated tests. Therefore, the fuzzy approach is well suited for such samples. Another advantage of the fuzzy logic is that all the rules are written verbally, much like human thought. Fuzzy logic allows the rules to be changed, membership functions to be modified as the way programmer wants to do. ANN models, on the other hand, are black box models including matrices of weights; numbers etc., and are not immediately visible to the user. Stock investors may easily adapt to the verbal rule creation process, rather than dealing with the numbers, and create their own fuzzy logic models.

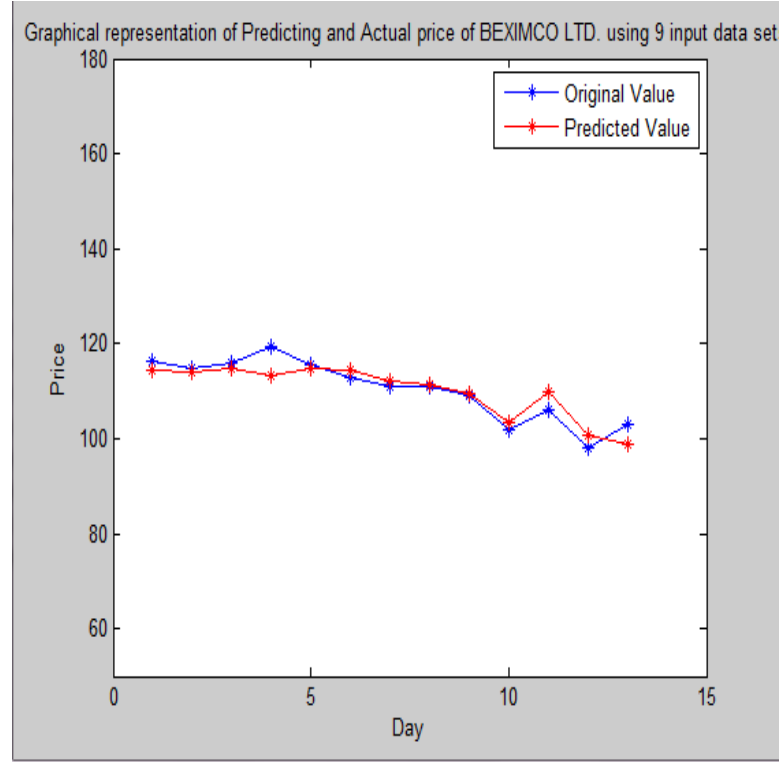

Fig 4: Graphical representation of Predicting and Actual price of BEXIMCO Ltd. using 9 input data sets [13]

\section{CONCLUTIONS}

As researchers and investors strive to out-perform the market, the use of fuzzy logic and neural networks to forecast stock market prices will be a continuing area of research. The ultimate goal is to increase the yield from the investment. It has been proven already through research that the evaluation of the return on investment in share markets through any of the traditional techniques is tedious, expensive and a time-consuming process. In conclusion we can say that if we create a strong knowledge base and train our system with more input data set it generate more error free prediction price.

\section{REFERENCES}

[1] White H, "Economic prediction using neural networks: The case of IBM daily stock returns". In Neural Networks in Finance and Investing, chapter 18, pages 315-328, 1993. Probus Publishing.

[2] Robert J. Van Eyden, "The Application of Neural Networks in the Forecasting of Share Prices". Finance and Technology Publishing, 1996.

[3] Samarth Agarwal, Manol Jindal, G.N.Pillai, "Momentum Analysis based Stock Market Prediction using ANFIS". In Proceeding of the International Multiconference of Engineering and Computer Scientists 2010 Vol.1, IMECS 2010, March 2010, Hong Kong.

[4] Rumelhart, D.D.m Hinton, G.E. and Williams, R.J., "Learning Internal Representation, Man, and Cybernetics (SMC'e91)”, 1991. 1913-1918.

[5] Simo Haykin, "Feedforward neural Networks: An Introduction", page (2-4).

[6] Stuart Russell, Peter Norvig, "Artificial Intelligence a modern approach", (second edition), 2004

[7] G. Fa-Liang, "A new way of predicting cement strengthfuzzy logic", Cement and Concrete Research 27, (1997) 883-888.

[8] J.-S. Roger Jang Ned Gulley "MATLAB ${ }^{\circledR}$ User's Guide Fuzzy Logic Version 1 Toolbox".

[9] Simon Haykin, "Neural Network A Comprehensive Foundation", second edition, Prentice Hall, 1998, page $161-173$

[10] Y.-Q. Zhang and A. Kandel, "Compensatory Genetic Fuzzy Neural Networks and Their Applications," Series in Machine Perception Artificial Intelligence, Volume 30, World Scientific, 1998.

[11] W.Duch and N. Jankowski, "Transfer functions: hidden possibilities for better neural networks.", 9th European Symposium on Artificial Neural Networks (ESANN), Brugge 2001. De-facto publications.

[12] "Stocks Basics: Introduction", http://www.investopedia.com/university/stocks/\#axzz1Y VyeGN1y.

[13] Y.T. Yu, M.F. Lau, "A comparison of MC/DC, MUMCUT and several other coverage criteria for logical decisions", Journal of Systems and Software, 2005, in press.

[14] Mark Hudson Beale Martin T. Hagan Howard B. Demuth "Neural Network Toolbox'TM 7 User's Guide". 